\title{
Transmissão e a influência do volume dos estoques públicos sobre o preço do arroz no Brasil
}

\author{
Transmission and influence of volume of public stocks on the price of rice in Brazil
}

\author{
Elisandra dos Santos ${ }^{\mathrm{I}}$ Laion Wolff $^{1}$ Adriano Mendonça Souza ${ }^{\mathrm{II}}$
}

\section{RESUMO}

\begin{abstract}
Este trabalho analisou o mecanismo de transmissão dos preços dos principais estados produtores de arroz no Brasil (Rio Grande do Sul, Mato Grosso e Santa Catarina), como variáveis endógenas, e volume dos estoques públicos no Brasil, como variável exógena, para o período de julho de 2004 até dezembro de 2010. A análise compreende o uso da metodologia VAR-VEC para identificar o número de defasagens utilizadas e verificar se existem co-integrações entre as variáveis, por meio do Teste de Co-integração de Johansen. Testou-se a existência de causalidade entre as séries pelo método de causalidade de Granger. Aplicou-se a decomposição da variância do erro de previsão e a função impulso-resposta com decomposição de Cholesky para analisar a relação entre os preços dos estados e volume em estoques públicos. Com a aplicação da metodologia proposta, pode-se observar que o preço do arroz no estado do Rio Grande do Sul influencia no preço no estado de Santa Catarina e no volume armazenado em estoques públicos e é influenciado pelo preço do arroz no estado do Mato Grosso.
\end{abstract}

Palavras-chave: transmissão de preços, estoque público, modelos de co-integração de Johansen, modelos autorregressivos com correção de erros, causalidade de Granger.

\section{ABSTRACT}

This study examined the mechanism of prices from major rice producing states in Brazil (Rio Grande do Sul, Mato Grosso and Santa Catarina), as endogenous variables, and volume of public stocks in Brazil, as exogenous variables for the period July 2004 until December 2010. The analysis includes the use of VAR-VEC methodology to identify the number of lags used and check for co-integration among variables through the test of Johansen Co-integration. We tested the existence of causality between variables by the method of Granger causality.
We applied the variance decomposition of forecast error and impulse-response function with Cholesky decomposition to analyze the relationship between prices and volume of the states in public stocks. With the proposed methodology, one can observe that the price of rice in the state of Rio Grande do Sul to the price in the state of Santa Catarina and the volume stored in stockpiles and is influenced by the price of rice in the state of Mato Grosso.

Key words: price of rice, stocks public autoregressive models with error correction, Granger causality.

\section{INTRODUÇÃO}

$\mathrm{O}$ arroz foi, possivelmente, o principal alimento e a primeira planta cultivada na Ásia. Há cerca de 5.000 anos, foram encontrados, na literatura chinesa, os registros mais antigos sobre ele. Muitos pesquisadores e especialistas assinalam o sudeste da Ásia como seu local de origem. Outros autores dizem que o cereal é originário do Japão, onde é cultivado há pelo menos 7 mil anos, ou da Índia, uma das regiões de maior variedade de arroz, sendo também, para alguns autores, centro de origem (EMBRAPA).

O Brasil é apontado por alguns historiadores como o país pioneiro a plantar esse cereal no continente americano. Conhecido como "milho d'água" (abatiuaupé), foi cultivado pelos índios muito antes de chegada dos Portugueses em 1500, nos alagados próximos ao litoral. Já em 1587, há registro de lavouras arrozeiras na Bahia e, mais tarde, aproximadamente em

'Universidade Federal de Santa Maria (UFSM),Santa Maria, RS, Brasil.

"Departamento de Estatística, UFSM, 97105-900, Santa Maria, RS, Brasil. E-mail: amsouza@ gmail.com. Autor para correspondência. 
1745, no Maranhão. A Coroa Portuguesa em 1766 autorizou a instalação da primeira descascadora de arroz no Brasil, na cidade do Rio de Janeiro, e a produção era voltada para a exportação do cereal.

Segundo VIEIRA \& OLIVEIRA (2011), o arroz encontra-se disseminado no mundo todo, sendo cultivado em todos os continentes, em cerca de 120 países, e o seu consumo pela população mundial é um hábito inquestionável. O Brasil figura entre os dez maiores produtores e consumidores no mundo, perdendo somente para China, Índia, Indonésia, Bangladesh, Vietnam, Tailândia e Mianmar.

O cereal possui grande valor econômico, é alimento básico para cerca de 2,4 bilhões de pessoas e, segundo estimativas, até 2050 , haverá uma demanda para atender ao dobro dessa população. Sua importância não é apenas alimentar, mas representa o maior potencial para o combate à fome no mundo. No Brasil, o arroz é um dos principais produtos da cesta básica e a população brasileira de baixa renda gasta metade da sua remuneração em alimentação e, desses $50 \%$, gasta $15 \%$ com arroz (EMBRAPA).

A formação do preço do arroz brasileiro depende de uma série de variáveis que mudam de estado para estado. Os fatores que influenciam no preço do arroz podem ser de ordem climática, logística (armazenagem, transporte, etc), mudança de cultura, oferta e demanda, êxodo rural, preço dos insumos e impostos, entre outros. O governo é o mediador do preço do arroz por meio de intervenções, em que procura manter a oferta do mercado em níveis desejados. Não é de hoje que o Brasil adota uma política de intervenção visando a estabelecer um equilíbrio entre a oferta e a procura. $\mathrm{O}$ mais famoso acordo foi $\mathrm{o}$ Convênio de Taubaté, em que o governo se comprometeu a adquir todo exedente de produção, visando a uma valorização do produto. Bem mais organizado e sem tendência de favorecimento particular, o governo adota uma política de proteção dos preços, em que mantém estoques públicos de grãos e utiliza esses estoques para manter o preço do produto competitivo no mercado nacional e internacional.

A posição contábil dos estoques públicos de arroz no Brasil em dezembro de 2010 era 61.148 mil toneladas, o que é considerado um estoque muito baixo para assegurar uma possível valorização do cereal. Segundo Sushil PANDEY (2008), economista agrícola do IRRI (International Rice Research Institute), uma das possíveis causas da diminuição do estoque é o desequilíbrio entre fornecimento e demanda, em longo prazo, o que vem ocorrendo há vários anos. Nos últimos três anos, houve longas estiagens na região sul do Brasil, propiciando uma quebra de produção de cerca de 40 milhões de toneladas do grão. Salienta-se que os estoques de cereais como o milho, trigo e arroz, que são a base da alimentação no mundo, estariam nos dias de hoje com reservas em $70 \%$ a menos do que estavam em 1999.

No intuito de contribuir para a literatura econômica agrícola, o trabalho tem como objetivo analisar a relação do preço da saca de arroz em casca entre os três principais estados produtores brasileiros, Rio Grande do Sul (RS), Mato Grosso (MT), Santa Catarina (SC), e o volume do estoque público nacional (VEPN), utilizando-se dos modelos vetoriais autorregressivos e dos modelos de correção de erros.

Para o cumprimento dos objetivos propostos, o trabalho está dividido da seguinte forma: na próxima seção, é feita a apresentação dos dados e a exposição dos métodos econométricos utilizados. $\mathrm{Na}$ terceira seção, é apresentada a metodologia empregada, bem como a apresentação da estratégia empírica para as relações entre os preços do arroz nos principais estados produtores com a cesta básica e com a quantidade em estoques públicos (Ton). A seção posterior traz os resultados obtidos com a aplicação da metodologia e, na última seção, são feitas as considerações finais sobre os resultados.

\section{MATERIAL E MÉTODOS}

Neste tópico, serão apresentadas as fontes de dados a serem utilizadas na análise empírica. Além da metodologia dos modelos de vetores autorregressivos (VAR), também se utilizam os modelos de correção de erros (VEC).

\section{Dados}

Os dados utilizados nesta pesquisa compreendem o período de julho de 2004 a dezembro de 2010 , referentes ao preço da saca de $50 \mathrm{~kg}$ do arroz em casca, com observações mensais, no Rio Grande do Sul (RS), no Mato Grosso (MT) e em Santa Catarina (SC), e ao volume de estoque público medido em toneladas (VEPN). A série de preço do arroz nesses três estados está disponível no site Instituto Riograndense de Arroz (www.irga.rs.gov.br/docs/ srcasca.pdf), com complemento do site da CEPA(Centro de Socioeconomia e Planejamento Agrícola), disponível em http://cepa.epagri.sc.gov.br/. A série do volume de arroz em estoque público armazenado, expressa em toneladas, foi obtida no site da CONAB (Companhia Nacional de Abastecimento), disponível em http:// www.conab.gov.br/OlalaCMS/uploads/arquivos/ 11_02_02_17_16_45_arroz.pdf). 
O tratamento estatístico e as estimações das equações foram realizadas no pacote estatístico Econometric EViews (versão 7.0). A análise proposta para identificar as inter-relações entre as variáveis será por meio da metodologia de correção de erros, em que, para a elaboração do modelo econométrico, seguem os seguintes procedimentos metodológicos:

Modelo econométrico

Inicialmente, realizam-se os testes de raízes unitárias para verificar a estabilidade das variáveis em estudo e utilizam-se o teste de Augmented Dickey-Fuller (ADF) (1979) e Kwaiatkowski, Phillips, Schmidt and Shin (KPSS) (1992), de modo a classificar a série em relação a sua estacionariedade. Deve-se observar que só é possível realizar um estudo de co-integração entre as variáveis se elas possuírem o mesmo grau de integração, isto é, ambas I(1) ou I(2) ou integração de ordem superior, de modo que exista um equilíbrio de longo prazo (SOUZA e MENEZES, 2011), estimando-se assim a modelagem de vetores de correção de erros (VEC).

Identificada a ordem de integração das variáveis, determinam-se os modelos de vetores autorregressivos (VAR) dessas variáveis. Para fins de análise, considere um sistema com $p$ variáveis independentes e relacionadas por uma memória autorregressiva:

$$
x_{t}=A_{0}+A_{i} \sum_{i=1}^{p} x_{t-i}+\varepsilon_{t}
$$

Sendo que $\mathrm{X}_{\mathrm{t}}$ é vetor das variáveis em estudo, $\mathrm{A}_{0}$ é a matriz de interceptos, $\mathrm{A}_{\mathrm{i}}, i=1,2, . . \mathrm{p}$ são matrizes com os termos das equações e $\varepsilon_{t}$ são os erros não correlacionados, com média zero e variância constante. Ainda, $\varepsilon_{t}$ é um vetor de choques não esperados nas variáveis em estudo.

Na construção do modelo VAR, é importante a identificação do número de defasagens a serem incluídas no modelo. Pois essas defasagens serão responsáveis pelos impactos de curto prazo e são determinados pelos critérios AIC (Akaike Information Criterion) e SBC (Schwarz Bayesian Criterion), de modo que as defasagens utilizadas forneçam o melhor modelo e as melhores estimativas futuras.

O próximo passo é verificar se há cointegração e o número de co-integrações existentes entre as variáveis, utilizando o método de Johansen, com o teste do traço $\left(\lambda_{\text {Traço }}\right)$ e do máximo autovalor $\left(\lambda_{\text {Max }}\right.$ ) (JOHANSEN \& JUSELIUS, 1990), de modo a verificar a existência de alguma combinação linear entre as variáveis. Conforme ENGLE \& GRANGER (1987), a definição de co-integração é de queeja $X_{\mathrm{t}}$ um vetor ( $N x 1$, os componentes de $X_{\mathrm{t}}$ são ditos co-integrados de ordem $(d, b)$, denotado por $\mathrm{x}_{\mathrm{t}} \sim \mathrm{CI}(d, b)$, se todos os componentes de $X_{\mathrm{t}}$ são $\mathrm{I}(d)$ ou existe um vetor, $\alpha \neq 0$ tal que $z_{t}=\alpha^{\prime} x_{t} \sim I(d-b), b>0$.

O teste de causalidade de GRANGER (1969) será utilizado com o intuito de verificar a relação causal entre as variáveis, em que se procura determinar, não apenas a direção de influência de cada variável, mas a existência de uma precedência temporal significativa, que irá influenciar no processo de previsão.

Os resultados possíveis do Teste de Causalidade de Granger são os seguintes:

i) Causalidade unidirecional de $\mathrm{X}$ para $\mathrm{Y}$ ou de $\mathrm{Y}$ para $\mathrm{X}$; ii) Causalidade bidirecional de $\mathrm{X}$ para $\mathrm{Y}$ e de $\mathrm{Y}$ para $X$; iii) Ausência de causalidade,ou seja, quando as variáveis $\mathrm{X}$ e Y são independentes, não existe uma relação de causalidade.

Dada a existência de co-integração, estimase o vetor de correção de erros (VEC), de modo a se determinar em que período as variáveis entrarão em equilíbrio de longo prazo (HARRIS, 1995). O modelo VEC pode ser escrito da seguinte forma:

$$
\Delta y_{t}=\Pi y_{t-p}+\Gamma_{1} \Delta y_{t-1}+\Gamma_{2} \Delta y_{t-2}+\ldots .+\Gamma_{p-1} \Delta y_{t-(p-1)}+\mu_{t}
$$

Em que $\mathrm{p}$ é o número de defasagens escolhidas no modelo VAR em uma etapa anterior. $\Pi=\beta^{\prime} \alpha$, em que $\beta$ é uma matriz ( $\mathrm{p} \times \mathrm{r}$ ), sendo em suas colunas os vetores de co-integração, e a é a matriz ( $\mathrm{p} \mathrm{x}$ r) contendo os coeficientes de ajustamento.

Esse modelo permite que se realizem as análises de impulso de resposta e decomposição de variância por meio de "choques" nas equações encontradas. A decomposição de variância do erro de previsão é um instrumento utilizado para descrever a dinâmica do sistema na abordagem VAR. Por esse método, torna-se possível identificar a proporção da variação total de uma variável devida a cada choque individual nas $k$ variáveis componentes do modelo.

\section{RESULTADOS E DISCUSSÃO}

Inicialmente, é observada a evolução das séries originais, o preço do arroz em saca de $50 \mathrm{~kg}$, nos três principais estados produtores brasileiros, e o volume armazenado em estoques públicos. Em relação ao preço da saca do arroz no estado do Mato Grosso, ele é superior ao dos outros dois estados na maioria do período em estudo e, no ano de 2008, o preço do arroz apresentou uma alta. Em contrapartida, o volume em estoques públicos apresenta uma queda. Segundo o Centro de Estudos Avançados em Economia Aplicada (CEPEA), dentre os fatores que afetaram o mercado nacional do arroz em casca em 2008 e fizeram desse um ano de preços elevados, estão o atraso na colheita e a baixa oferta do produto no início do ano. Nos primeiros 
meses, apesar dos leilões de venda da Conab e da entrada do produto no mercado, os produtores continuaram recuados em função do aumento nos custos de produção. No último bimestre, os preços caíram, devido à dificuldade de repasse do preço do arroz em casca ao beneficiado e à retração do setor atacadista/varejista.

Como proposto, para avaliar a estacionariedade das séries, foi realizado o teste de estacionariedade por meio do teste de $\mathrm{ADF}$, que possui a hipótese $\mathrm{H}_{0}$ de que a série possui raiz unitária $\mathrm{I}(1)$, isto é, a série não é estacionária. Não há evidências suficientes para se rejeitar a hipótese nula, logo, a série é classificada como não estacionária em nível. Quando estas séries são analisadas em primeiras diferenças, rejeita-se a hipótese nula, logo há ausência de raiz unitária, pode-se dizer que as séries são estacionárias em primeiras diferenças.

Com o objetivo de confirmar os resultados obtidos pelo teste ADF, empregou-se o teste KPSS, que tem como hipótese nula a estacionariedade da série. O teste de KPSS inverte a hipótese nula (estacionariedade) em relação à hipótese alternativa de existência de raiz unitária. De acordo com KWIATKOWSKI et al. (1992, p. 176), o teste KPSS tende a complementar o teste de raiz unitária de DickeyFuller. Observa-se, nesse caso, que, em nível, não se pode rejeitar a hipótese alternativa de não estacionariedade das séries, enquanto que, depois de aplicada uma diferença, pode-se dizer que as séries são estacionárias.

Os resultados indicam que todas as séries são estacionárias em primeira diferença, tanto pelo teste ADF como KPSS, com a hipótese de não estacionariedade, sendo rejeitada ao nível de significância de 5\% para todas as variáveis.

No Brasil, o período de colheita do arroz (chamada safra) se dá no período de março a junho, ocorrendo assim uma grande oferta do produto. No período de agosto a dezembro, ocorre a entressafra da cultura (CONAB) e há uma oscilação dos preços praticados que acompanham a safra e a entressafra, refletindo na não estacionariedade das séries em estudo, de modo que é possível que haja um equilíbrio de longo prazo. Dado que as séries são não estacionárias, acompanhando o período de safra e entre safra, é necessário estimar um modelo VAR para se avaliar a dinâmica de curto prazo e, com isso, determinar o número de defasagens incluídas no VAR para estimar estas relações entre as variáveis. Nesse processo, são testados diferentes números de defasagens, sendo que o menor valor dos critérios AIC e SBC foi obtido ajustando o modelo com 1 (uma) defasagem para cada variável.
Definido o número de defasagens, podemos avaliar a presença de vetores de co-integração pelo método de Johansen, em que as relações de cointegração das variáveis foram definidas para o modelo VAR com 1 defasagem. Os resultados indicam a estatística do máximo autovalor e do traço, em que se observa que se rejeita a hipótese nula de $\mathrm{r}=0$ (não existe nenhum vetor de co-integração) ao nível de significância de 5\%. Logo, existe pelo menos um vetor de co-integração entre as séries estudadas.

Constatada a presença de relações de cointegração entre as séries em estudo, parte-se, então, para a construção de um modelo que leve em consideração essa relação de elasticidade de curto e longo prazo por meio de um modelo de Vetor de Correção de Erros (VEC). Os coeficientes do modelo de vetores autorregressivos com correção de erro (VEC) ajustado para explicar o comportamento do preço no RS, que se mostraram significativos, foram o volume dos estoques públicos e o preço do arroz no MT e em SC. Pode-se denotar que um aumento de $1 \%$ no preço do arroz no estado do MT e em SC gera uma variação de $0,17 \%$ e $0,65 \%$ no preço do estado do RS, respectivamente. Mesmo sendo significativo, o VEPN tem uma variação percentual muito pequena, pois o volume presente nos estoques não influencia no preço, mas sim a intervenção do governo no mercado que significa uma forma de regular o preço.

A partir do modelo estimado, podemos determinar as relações de causalidade existentes entre as séries em estudo para o modelo VAR/VEC em primeiras diferenças com uma defasagem. Esse estudo é realizado por meio do teste de Causalidade de Granger, que possibilita identificar que o preço do arroz em casca produzido no MT causa o preço do arroz no RS e em SC. Também o preço do arroz no RS tem uma relação de causalidade com o preço do arroz praticado em SC, o que já era esperado, pois o preço do arroz no MT é historicamente superior ao dos outros estados, assim como o preço no RS é superior ao do estado de SC. Ainda podemos notar que os três estados (MT, SC, RS) causam o VEPN em toneladas armazenadas, uma vez que esses estados são os principais produtores de arroz. Além disso, o volume dos estoques públicos causa o preço do arroz nos estados de MT e SC.

A comercialização do arroz no Brasil geralmente ocorre de duas maneiras, a primeira na venda à vista na época da colheita, o que favorece uma comercialização do produto a um preço baixo. A segunda na estocagem em cooperativas à espera da entressafra, de modo a garantir um preço de comercialização mais elevado ADAMI e MIRANDA (2011). A relação bidirecional de causalidade dos preços praticados entre 
RS e SC deve-se às características de plantio. Nos estados do Sul, produz-se um arroz em zonas denominadas de Várzeas que exigem grande quantidade de água e esta cultura já está estabelecida e arraigada à cultura sulina. No MT, produz-se o arroz sequeiro, com características e tecnologias diferenciadas de produção e onde a cultura está buscando uma estabilização, pois concorre com culturas como soja e algodão. De acordo com ADAMI et al. (2007), os formadores de política para a comercialização dessa cultura não seguem a flutuação dos preços, mas a garantia de estoques mínimos, denominados de nível mínimo de referência. Por essa razão, vê-se a relação de causalidade entre os estoques públicos e os estados produtores. Embora haja a intervenção do governo de modo a garantir os estoques mínimos e indiretamente o preço do produto, a oscilação na produção da cultura tem mantido as flutuações dos preços de venda.

Sendo observadas todas as relações existentes, o próximo procedimento realizado foi a análise da decomposição da variância do erro de previsão. Na análise do preço do arroz no RS, a decomposição de variância mostrou que, decorridos três meses, a variável que representa o preço no estado explica 93,23\% dela mesma, enquanto que é explicada pelo preço no MT em apenas 5,16\%. Em relação à decomposição de variância para MT, após três meses, mostrou que ela própria se explica $53,13 \%$, enquanto que o RS explica 45,79\% de MT. Já a variável que denota o preço do arroz em SC ressalta que, em três meses decorridos, ela é explicada em $73,1 \%$ pelo RS e apenas $23,5 \%$ por ela própria. No que se refere à decomposição de variância para o volume armazenado em estoque público, observa-se que $68,4 \%$ é explicado por ela mesma, enquanto que 16,6\% é explicada pelo RS.

Por meio dos resultados encontrados na estimação do modelo vetorial autorregressivo com correção de erros, foram analisadas as funções de impulso-resposta que demonstram os efeitos sobre o preço do arroz no RS a choques nas outras variáveis do modelo, para os 75 meses seguintes. Com os gráficos do impulso-resposta (Figura 1), é possível verificar o impacto que um choque de um desvio-padrão em uma série (impulso) causa no desvio-padrão da outra série (resposta). Quando administrado um choque no desviopadrão da variável que denota o preço do arroz no MT, observa-se que o preço no RS oscila positivamente por volta dos 50 meses, com um índice negativo dos 25 aos 50 meses e, a partir daí, permanece estável.

Em relação ao impulso no desvio-padrão da variável que representa o preço do arroz em SC e no desvio-padrão da variável que demonstra a quantidade de arroz em estoques públicos, há uma resposta no desviopadrão do preço no RS muito pequena. Quanto a impulsos administrados no desvio-padrão da variável que evidencia o volume em estoques públicos, observa-se que há uma variação positiva em torno de 30 meses no preço do arroz do RS, enquanto que, dos 30 aos 65 meses, essa variação é negativa e, a partir de 65 meses, é estável.

\section{CONCLUSÃO}

Nesta pesquisa, buscou-se determinar a relação de transmissão de preço do arroz entre os principais estados produtores brasileiros (Rio Grande Sul, Mato Grosso e Santa Catarina). Além disso, pretendeu-se verificar a influência do volume de toneladas de arroz armazenadas em estoques públicos sobre o preço do arroz nestes estados. Salienta-se que há uma relação de equilíbrio de longo prazo, envolvendo tanto os estados produtores em análise como as intervenções realizadas pelo governo para garantir o estoque mínimo de garantia. Dessa forma, analisaramse as relações de causalidade entre as variáveis por

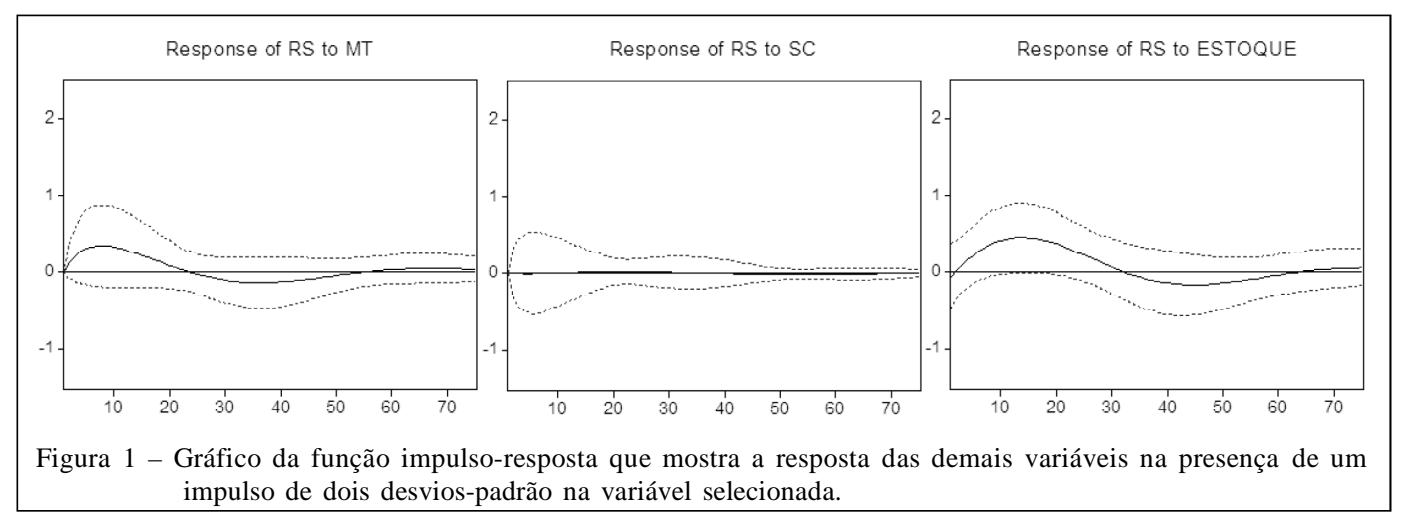

Ciência Rural, v.43, n.3, mar, 2013. 
meio do teste de Causalidade de Granger, em que o preço do arroz no estado do Rio Grande do Sul causa "Granger" o preço no estado de Santa Catarina. O volume armazenado em estoques públicos e o preço do arroz no Mato Grosso causam "Granger" o preço no Rio Grande do Sul e Santa Catarina. Também foi avaliado o percentual de variação de cada variável sobre as demais e sobre ela mesma, pelo método da decomposição de variância, em que foi possível verificar que, após decorridos três meses, o percentual de explicação das variáveis por ela mesma são de: $93,22 \%$ para o preço no RS, $53,14 \%$ para o MT, $23,46 \%$ para SC, $68,41 \%$ para o volume em estoques públicos. Pela avaliação da função impulso-resposta, sendo a resposta o preço no Rio Grande do Sul, pode-se perceber que um impulso nas demais variáveis causa uma variação, sendo que, em alguns pontos, é positiva e, em outros, negativa, e a estabilidade é alcançada por volta de 60 meses.

Assim sendo, com este trabalho, foi possível verificar que há uma transmissão do preço do arroz entre os estados e os VEPN de modo que políticas públicas e estratégias de comercialização devem ser tomadas com cautela, pois o seu efeito será transmitido no longo prazo. O estudo então possibilita a verificação do tempo decorrido para que estas intervenções governamentais e equilíbrio de longo prazo venham a ocorrer.

\section{AGRADECIMENTOS}

Aos revisores anônimos pela valiosa contribuição dada a este texto. Também ao suporte financeiro da Coordenação de Aperfeiçoamento de Pessoal de Nível Superior (CAPES) e ao Laboratório de Análise e Modelagem Estatística - DE- UFSM, para a realização desta pesquisa.

\section{REFERÊNCIAS}

ADAMI, A.C.O.; MIRANDA, S.H.G. Transmissão de preços e cointegração no mercado brasileiro de arroz. Revista de Economia e Sociologia Rural, v.49, n.1, p.55-80, 2011.

CEPA (CENTRO DE SOCIOECONOMIA E PLANEJAMENTO AGRÍCOLA). Disponível em: 〈http://cepa.epagri.sc.gov.br〉. Acesso em: 26 fev. 2011.
CONAB (COMPANHIA NACIONAL DE ABASTECIMENTO). Disponível em: <http://www.conab.gov.br/OlalaCMS/uploads/arquivos/ 11_02_02_17_16_45_arroz.pdf>. Acesso em: 12 mar. 2011.

DICKEY, D.A.; FULLER, W.A. Distribution of the estimator for auto-regressive time series with a unit root. Journal of the American Statistical Association, v.74, p.427-431, 1979.

EMBRAPA. Ministério da Agricultura, Pecuária e Abastecimento. Arroz e feijão. Disponível em: <http://www.embrapa.br/ kw_storage/keyword.2007-07-19.4200885731>. Acesso em: 25 fev. 2011.

ENGLE, F.; GRANGER, J. Co-integration and error correction: Representation, estimation and testing. Econometrica, v.2, p.251-276, 1987.

GRANGER, C.W. Investigating casual relations by econometric models and cross spectral methods. Econometrica, v.37, p.424$438,1969$.

HARRIS, R.I.D. Cointegration analysis in econometric modelling. London: Prentice Hall, 1995. 176p.

IRGA (INSTITUTO RIO GRANDENSE DE ARROZ). Disponível em: 〈http://www.irga.rs.gov.br/docs/srcasca.pdf〉. Acesso em: 15 mar. 2011.

JOHANSEN, S.; JUSELIUS, K. Maximum likelihood estimation and inference on cointegration - with aplications to the demand for money. Oxford Bulletin of Economics and Statistics, v.52, p.169-219, 1990.

KWIATKOWSKI, D.P.C.B. et al. Testing the null hypothesis of stationarity against the alternative of a unit root: how sure are we that economic time series have a unit root? Journal of Econometrics, v.54, p.159-178, 1992.

PANDEY, S. (International Rice Research Institute ). Aumento no preço do arroz deve continuar, diz instituto BBC Brasil. O Globo, Rio de Janeiro, 11 de abr. 2008. Disponível em: <http:/ /oglobo.globo.com/economia/mat/2008/04/11/aumento no_preco_do_arroz_deve_continuar_diz_instituto-4268001 65.asp>. Acesso em: 19 mar. 2011.

SOUZA, A.M.; SOUZA, F. MENEZES,R. Analysis of equilibrium in industrial variables through error correction models. International Journal of Aacademic Rresearch, v.3, n.1, p.359-364, 2011.

VIEIRA, N.R.A.; OLIVEIRA, M.A.S. Arroz. In: EMBRAPA: Ministério da Agricultura, Pecuária e Abastecimento. Disponível em: 〈http://www.agroplan-consultoria.com.br/historia_arroz/>. Acesso em: 8 mar. 2011. 